\title{
Statistical mechanics of neocortical interactions Large-scale EEG influences on molecular processes
}

\author{
Lester Ingber ${ }^{\mathrm{a}, 1}$ \\ ${ }^{a}$ Lester Ingber Research, Ashland, OR
}

\begin{abstract}
Calculations further support the premise that large-scale synchronous firings of neurons may affect molecular processes. The context is scalp electroencephalography (EEG) during short-term memory (STM) tasks. The mechanism considered is $\boldsymbol{\Pi}=\mathbf{p}+q \mathbf{A}$ (SI units) coupling, where $\mathbf{p}$ is the momenta of free $\mathrm{Ca}^{2+}$ waves $q$ the charge of $\mathrm{Ca}^{2+}$ in units of the electron charge, and $\mathbf{A}$ the magnetic vector potential of current $\mathbf{I}$ from neuronal minicolumnar firings considered as wires, giving rise to EEG. Data has processed using multiple graphs to identify sections of data to which spline-Laplacian transformations are applied, to fit the statistical mechanics of neocortical interactions (SMNI) model to EEG data, sensitive to synaptic interactions subject to modification by $\mathrm{Ca}^{2+}$ waves.
\end{abstract}

Keywords: short-term memory, astrocytes, neocortical dynamics, vector potential

\section{Introduction to top-down premise (TDP)}

This project is motivated by a top-down premise (TDP) that large-scale synchronous neural firings, as measured by scalp electroencephalographic recordings (EEG), may directly influence molecular processes that are coupled to underlying synaptic processes that contribute to this synchrony (Ingber, 2011, 2012, 2015; Ingber et al., 2014; Nunez et al., 2013).

Previous papers (Ingber, 2015; Ingber et al., 2014) have primarily been concerned with fitting EEG data to the author's statistical mechanics of neocortical interactions (SMNI) model (Ingber, 1982, 1983) to study top-down largescale synchronous neuronal firings during short-term memory (STM) tasks, also modeled by SMNI (Ingber, 1984, 1985a, 1994), on coupled bottom-up molecular processes.

Only for brevity, unless otherwise stated, dependent on the context, "EEG" will refer to either the measurement of synchronous firings large enough to measurable on the scalp, or to the firings themselves. The term "molecular processes" is used to signify not only the scale included, but also the effects of $\mathrm{Ca}^{2+}$ waves on synaptic background molecular processes that ultimately drive neuronal firings via releases of quanta of chemical transmitters, e.g., affecting molecules of phospholipid bilayers at presynaptic neuronal membrane sites. "Top-down" refers the largest scale considered of highly synchronous neuronal firings as measured by EEG, and "bottom-up" refers to the smallest scale considered here of $\mathrm{Ca}^{2+}$ waves as they affect background synaptic processes that contribute to the neuronal firings measured by EEG in the context of STM tasks.

Free regenerative $\mathrm{Ca}^{2+}$ waves, arising from astrocyte-neuron interactions, couple to the magnetic vector potential $\mathbf{A}$ produced by these collective firings. As calculated in these papers, only $\mathbf{A}$, not electric $\mathbf{E}$ or magnetic $\mathbf{B}$ fields have logarithmic insensitivity from their sources to add cumulatively to affect the molecular processes. Calculations described here, using data processsed for proper matching to the SMNI model, give more support to TDP.

This study sheds light on the multiple scales of neocortical interactions underlying Consciousness (C). It is now accepted by some neuroscientists and confirmed by some experiments (Asher, 2012; Salazar et al., 2012), that at least

\footnotetext{
Email address: ingber@alumni.caltech.edu (Lester Ingber)

URL: http: //www . ingber . com (Lester Ingber)

${ }^{1}$ Corresponding Author
} 
some memories are actively processed by highly synchronized patterns of neuronal firings as measured by scalp EEG recordings during activity of processing such patterns, e.g., P300 waves, etc. This was always the basic premise of the SMNI model.

Without detailed experimental testable facts on the nature of $\mathbf{C}$, many people consider TDP as just conjecture on other future theories of $\mathbf{C}$. As discussed in a previous paper (Ingber, 2015) there are aspects of $\mathbf{C}$ that we may only be able to infer existence or possibly prove we cannot know. These latter possibilities can be considered as belonging to a "Dark C" (DC) category, and DC should be researched as well as C.

There are many other people that consider $\mathbf{C}$ as an emergent property of a complex system, and in this context TDP is a strong indicator of multiple scales of neocortical interactions underlying $\mathbf{C}$.

There are many papers that deal directly with or give credence to advances in $\mathbf{C}$ that include quantum mechanics, that have passed a sometimes volatile peer-review process in this subject in various physics journals (Zhou et al., 2015), which has led to some critical reviews (McKemmish et al., 2009). A current summary gives fair credit to the new ideas presented (Atmanspacher, 2015).

However, this study demonstrates how models of $\mathbf{C}$ can be developed faithful to experimental data. The scientific focus on computational models that include experimental data opens these ideas to testable hypotheses.

Results of SMNI fits to EEG data gave strong confirmation of the SMNI model of STM, and now also give weak statistical support to a basic physical mechanism that couples highly synchronous firings to control underlying molecular processes, the canonical momentum $\boldsymbol{\Pi}, \boldsymbol{\Pi}=\mathbf{p}+q \mathbf{A}$, where $\mathbf{p}$ is the momenta of a $\mathrm{Ca}^{2+}$ wave, $q$ the charge of $\mathrm{Ca}^{2+}, q=-2 e, e$ the magnitude of the charge of an electron.

Previous papers have used classical physics to calculate and compare the molecular $\mathbf{p}$ and large-scale q $\mathbf{A}$ components of $\boldsymbol{\Pi}$, demonstrating that indeed they of comparable magnitudes (Ingber, 2011, 2012; Ingber et al., 2014; Nunez et al., 2013). Also, in the context of quantum mechanics, the wave function of the $\boldsymbol{\Pi}$ system was calculated, and it was demonstrated that overlap with multiple collisions during the observed long durations of typical $\mathrm{Ca}^{2+}$ waves (Ingber, 2015; Ingber et al., 2014) support a Zeno effect (Facchi et al., 2004; Facchi and Pascazio, 2008; Giacosa and Pagliara, 2014; Kozlowski et al., 2015; Patil et al., 2015; Wu et al., 2012; Zhang et al., 2014) promoting long coherence times. This approach also suggests some nanosystem-pharmaceutical applications (Ingber, 2015).

Note that this proposed quantum context of the $\Pi$ interaction does not depend on any quantum effects via internal energy levels, for which $\mathrm{Ca}^{2+}$ with atomic weight 40 likely would not support at room temperatures (Beck, 2008).

Much of the theoretical development in this paper concerns calculations that support the influence of EEG on $\mathrm{Ca}^{2+}$ waves, and referencing experimental support of how $\mathrm{Ca}^{2+}$ waves affect background synaptic noise. However, the actual fits to EEG data, comprising several CPU-years of calculations on parallel-processor supercomputers, concerns the importance of the background synaptic noise that includes functional dependence on widespread regional synchrony arising from logarithmic insensitivity of $\mathbf{A}$ to distance from minicolumnar currents. This functional dependence is in the sole argument of the probability distribution of quanta release which directly influences molecular processes underlying this EEG.

\section{Theory}

\subsection{Molecular processes contributing to synaptic interactions}

There are many studies on tripartite neuron-astrocyte interactions (Pereira and Furlan, 2009), and on $\mathrm{Ca}^{2+}$ waves at tripartite sites. The short summary below is presented to set the context for SMNI calculations of probability distributions of synaptic activity that include background contributions.

Several studies have shown that glutamate release from astrocytes through a $\mathrm{Ca}^{2+}$-dependent mechanism can activate receptors located at the presynaptic terminals. Regenerative intercellular calcium waves (ICW) can travel over 100's of astrocytes, encompassing many neuronal synapses. These ICWs are documented in the control of synaptic activity (Scemes and Giaume, 2006). Analysis of fluorescence accumulation clearly demonstrate that glutamate is released in a regenerative manner, with subsequent cells that are involved in the calcium wave releasing additional glutamate (Innocenti et al., 2000).

Several experiments, albeit with sometimes contradicting conclusions, support astrocyte generated $\left[\mathrm{Ca}^{2+}\right]([\ldots]$ $=$ concentration $)$ influences on synapses (Volterra et al., 2014). They conclude that new experimental paradigms are 
required to address external interferences, e.g., possible interference of measurement indicators, etc., wherein $\left[\mathrm{Ca}^{2+}\right]$ affect increased release probabilities at synaptic sites, likely due to triggering release of gliotransmitters. It has been noted by other investigators that intracellular astrocyte calcium waves in situ (not the free $\mathrm{Ca}^{2+}$ waves considered here), increases neuronal firings (Fiacco and McCarthy, 2004), and that $\mathrm{Ca}^{2+}$ waves in general may have various influences on neuronal firings (Agulhon et al., 2008). It also has been suggested that by simultaneously activating and deactivating neurotransmission in all of the synapses enveloped by an astrocyte, the astrocyte calcium wave may coordinate synapses into synchronously firing groups (Mitterauer and Kofler-Westergren, 2011).

Although the full set of mechanisms affecting $\left[\mathrm{Ca}^{2+}\right]$ and the influences of $\mathrm{Ca}^{2+}$ on other mechanism are not yet fully understood and experimentally verified, it is clear that $\mathrm{Ca}^{2+}$ waves exist in intercellular as well as in intracellular media (Ross, 2012). There are regenerative as well as non-regenerative processes observed, both "locally" at cellular sites as well as into "expanded" regions through which $\mathrm{Ca}^{2+}$ travel at relatively fast velocities for large distances over relatively long periods of time (Ingber, 2015; Ingber et al., 2014). $\mathrm{Ca}^{2+}$ affects spontaneous synaptic production of glutamate, in contrast to also possibly influencing evoked production due to neuronal firings.

\subsection{Statistics of synaptic interactions}

The probability of a neuron firing, in the context of the previous Section, is based on the statistics of quantal releases of chemical neurotransmissions across synaptic gaps. This probability can be determined by a folding of two distributions, a distribution of quantal release of neurotranmitters across a given synapse, $\Psi$, and a distribution over all synapses and the properties of neuron that affect its firing or not firing, $\Gamma$ (discussed in the next Section).

As calculated previously (Ingber, 1982, 1983), the interaction of neuron $k\left(k=1, N^{*}\right)$ with neuron $j$ across all $j k$ synapses is defined by a distribution $\Psi$ describing $q$ chemical quanta with mean efficacy

$$
a_{j k}^{*}=\frac{1}{2} A_{j k}^{*}\left(\sigma_{k}+1\right)+B_{j k}^{*} \approx 0.01
$$

with $B_{j k}^{*}$ a background spontaneous contribution, where $\sigma_{k}=1$ if $k$ fires; $\sigma_{k}=-1$ if $k$ does not fire. Synaptic efficacy is a measure of ionic permeability, and an inverse measure of electrical impedance. As detailed previously (Ingber, 1982, 1983), efficacies $A_{j k}^{*}$ measure chemical synaptic activity, while efficacies $B_{j k}^{*}$ measure background influences. It was shown that the final results of folding $\Gamma$ and $\Psi$ distributions is independent of choosing $\Psi$ to be Gaussian or Poisson (Ingber, 1982, 1983), generalizing earlier work (Shaw and Vasudevan, 1974).

For example, if $\Psi$ is Poisson,

$$
\Psi=\exp \left(-a_{j k}^{*}\right)\left(a_{j k}^{*}\right)^{q} / q !
$$

This $\Psi$ is essentially the probability discussed in papers referenced in the previous Section, due to triggering release of gliotransmitters, which may be influenced by $\mathrm{Ca}^{2+}$ waves arising from neuron-astrocyte tripartite interactions. $B_{j k}^{*}$ is the variable that will be considered a function of $\mathbf{A}$ below, i.e., the TDP mechanism detailing how top-down activity measured by EEG during STM tasks may influence molecular $\mathrm{Ca}^{2+}$ waves. E.g., $\mathrm{Ca}^{2+}$ waves can control glutumate production which can control the distribution of $q$ quanta released at synaptic gaps. This is consistent with $\mathrm{Ca}^{2+}$ waves from astrocytes as contributing a diffuse control of neuronal firings via $B_{j k}^{*}$, rather than the more direct control of $A_{j k}^{*}$ due to presynaptic firings.

\subsection{Scale to neuronal firing}

A Gaussian distribution $\Gamma$ describes the average intra-neuronal distribution of electrical polarization across the various neurons in a minicolumn,

$$
\begin{gathered}
\Gamma=\left(2 \pi q \phi_{j k}^{2}\right)^{-1 / 2} \exp \left[-\left(W_{j k}-q v_{j k}\right)^{2}\right] /\left(2 q \phi_{j k}^{2}\right), \\
\lim _{q \rightarrow 0} \Gamma \equiv \delta\left(W_{j k}\right),
\end{gathered}
$$

where parameters in $\Gamma$ are specified below. 
Using the probability of developing potential $W_{j k}$ from $k$,

$$
S_{j k}=\sum_{q=0}^{\infty} \Gamma \Psi
$$

and the probability $S_{j}$ of developing $W_{j k}$ from all afferent neurons

$$
S_{j}=\int \ldots \int d W_{j 1} \ldots d W_{j N^{*}} S_{j 1} \ldots S_{j N^{*}} \delta\left(W_{j}-\sum_{k} W_{j k}\right)
$$

the derived probability for neuron $j$ to fire, given its interaction with $k=1, \ldots, N^{*}$ neurons is (Ingber, 1982, 1983)

$$
\begin{gathered}
p_{\sigma_{j}}=\int_{V_{j}}^{\infty} d W_{j} S_{j} \simeq \exp \left(-\sigma_{j} F_{j}\right) /\left[\exp F_{j}+\exp \left(-F_{j}\right)\right] \\
F_{j}=\frac{V_{j}-\sum_{k} a_{j k^{*}}^{*} v_{j k}}{\left((\pi / 2) \sum_{k^{\prime}} a_{j k^{\prime}}^{*}\left(v_{j k^{\prime}}^{2}+\phi_{j k^{\prime}}^{2}\right)\right)^{1 / 2}}
\end{gathered}
$$

Note the dependence of $F_{j}$ on synaptic parameters which will be discussed again below.

\subsection{Scale to mesocolumnar neuronal interactions}

Mesocolumns are defined as the dynamics of divergence from minicolumnar processes, and convergence to regional and macrocolumnar processes. Statistical mechanics of neocortical interactions (SMNI) was developed to explicitly model these dynamics (Ingber, 1982, 1983). The math requires a nonlinear stochastic calculus, first formulated within the context of gravity by many authors (Cheng, 1972), and subsequently generalized to other classical physics systems by many other authors (Langouche et al., 1982). At this stage SMNI is a zero-fit-parameter theory, in that all parameters are picked from within experimentally determined ranges. However, three basic models were then developed with slight adjustments of the parameters (Ingber, 1984), changing the firing component of the columnaraveraged efficacies $A_{j k}$ within experimental ranges as discussed below.

Using this theory as a guide, discoveries were made that indeed modeled various aspects of neocortical interactions, e.g., properties of STM -- e.g., capacity (auditory $7 \pm 2$ and visual $4 \pm 2$ ), duration, stability, primacy versus recency rule, Hick's law, nearest-neighbor minicolumnar interactions within macrocolumns calculating rotation of images, etc (Ingber, 1982, 1983, 1984, 1985a, 1994). SMNI was also scaled to include mesocolumns across neocortical regions to fit EEG data, as it used here as well (Ingber, 1997a, 2012).

The resulting mathematics is used here for SMNI modeling of EEG data, further generalized to include possible interactions with $\mathrm{Ca}^{2+}$ molecular processes. Using $G=\{E, I\}$ to represent independent excitatory $E$ and inhibitory $I$ processes, in the prepoint (Ito) representation the SMNI Lagrangian $L$ is

$$
\begin{gathered}
L=\sum_{G, G^{\prime}}(2 N)^{-1}\left(\dot{M}^{G}-g^{G}\right) g_{G G^{\prime}}\left(\dot{M}^{G^{\prime}}-g^{G^{\prime}}\right) /(2 N \tau)-V^{\prime} \\
g^{G}=-\tau^{-1}\left(M^{G}+N^{G} \tanh F^{G}\right) \\
g^{G G^{\prime}}=\left(g_{G G^{\prime}}\right)^{-1}=\delta_{G}^{G^{\prime}} \tau^{-1} N^{G} \operatorname{sech}^{2} F^{G} \\
g=\operatorname{det}\left(g_{G G^{\prime}}\right)
\end{gathered}
$$

where $N^{G}=\left\{N^{E}=160, N^{I}=60\right\}$ was chosen for visual neocortex, $\left\{N^{E}=80, N^{I}=30\right\}$ was chosen for all other neocortical regions, $M^{G^{\prime}}$ and $N^{G^{\prime}}$ in $F^{G}$ are afferent macrocolumnar firings scaled to efferent minicolumnar firings by 
$N / N * \approx 10^{-3}$, and $N *$ is the number of neurons in a macrocolumn, about $10^{5} . \tau$ is usually considered to be on the order of 5-10 msec; this is further discussed below in the Section below on coarse-graining EEG data.

Moving averages of several epochs of $g^{G}$ are used as slower drifts as described below in the data Section.

The threshold factor $F^{G}$ is derived as

$$
\begin{gathered}
F^{G}=\sum_{G^{\prime}} \frac{v^{G}+v^{\ddagger E^{\prime}}}{\left((\pi / 2)\left[\left(v_{G^{\prime}}^{G}\right)^{2}+\left(\phi_{G^{\prime}}^{G}\right)^{2}\right]\left(\delta^{G}+\delta^{\ddagger E^{\prime}}\right)\right)^{1 / 2}} \\
v^{G}=V^{G}-a_{G^{\prime}}^{G} v_{G^{\prime}}^{G} N^{G^{\prime}}-\frac{1}{2} A_{G^{\prime}}^{G} v_{G^{\prime}}^{G} M^{G^{\prime}} \\
v^{\ddagger E^{\prime}}=-a_{E^{\prime}}^{\ddagger E} v_{E^{\prime}}^{E} N^{\ddagger E^{\prime}}-\frac{1}{2} A_{E^{\prime}}^{\ddagger E} v_{E^{\prime}}^{E} M^{\ddagger E^{\prime}} \\
\delta^{G}=a_{G^{\prime}}^{G} N^{G^{\prime}}+\frac{1}{2} A_{G^{\prime}}^{G} M^{G^{\prime}} \\
\delta^{\ddagger E^{\prime}}=a_{E^{\prime}}^{\ddagger E} N^{\ddagger E^{\prime}}+\frac{1}{2} A_{E^{\prime}}^{\ddagger E} M^{\ddagger E^{\prime}} \\
a_{G^{\prime}}^{G}=\frac{1}{2} A_{G^{\prime}}^{G}+B_{G^{\prime}}^{G}, a_{E^{\prime}}^{\ddagger E}=\frac{1}{2} A_{E^{\prime}}^{\ddagger E}+B_{E^{\prime}}^{\ddagger E}
\end{gathered}
$$

where $\left\{A_{G^{\prime}}^{G}, B_{G^{\prime}}^{G}, A_{E^{\prime}}^{\ddagger E}, B_{E^{\prime}}^{\ddagger E}\right\}, A_{G^{\prime}}^{G}$ is the columnar-averaged direct synaptic efficacy, $B_{G^{\prime}}^{G}$ is the columnar-averaged backgroundnoise contribution to synaptic efficacy. $A_{G^{\prime}}^{G}$ and $B_{G^{\prime}}^{G}$ have been scaled by $N * / N \approx 10^{3}$ to keep $F^{G}$ invariant. Other values taken are consistent with experimental data, e.g., $V^{G}=10 \mathrm{mV}, v_{G^{\prime}}^{G}=0.1 \mathrm{mV}, \phi_{G^{\prime}}^{G}=0.03^{1 / 2} \mathrm{mV}$. The “‡”, parameters arise from regional interactions across many macrocolumns.

Note that the mesoscopic threshold funcion $F^{G}$ has similar functional dependencies on neuronal parameters as the individual threshold function $F_{j}$. This provides an audit trail back to neuronal parameters when $F^{G}$ is used in further scaling to regional dynamics to fit scalp EEG data.

\subsubsection{Centering Mechanism (CM)}

As mentioned above, three basic models were developed with slight adjustments of the parameters (Ingber, 1984), changing the firing component of the columnar-averaged efficacies $A_{G^{\prime}}^{G}$ within experimental ranges, which modify $F^{G}$ threshold factors to yield (a) case EC, dominant excitation subsequent firings in the conditional probability, or (b) case IC, inhibitory subsequent firings, or (c) case BC, balanced between EC and IC. Furthermore, a Centering Mechanism (CM) on case $\mathrm{BC}$ yields case $\mathrm{BC}^{\prime}$ wherein the numerator of $F^{G}$ only has terms proportional to $M^{E^{\prime}}, M^{I^{\prime}}$ and $M^{\ddagger E^{\prime}}$, i.e., zeroing other constant terms by resetting the background parameters $B_{G^{\prime}}^{G}$, still within experimental ranges. This has the net effect of bringing in a maximum number of minima into the physical firing $M^{G}$-space. The minima of the numerator then defines a major parabolic trough,

$$
A_{E}^{E} M^{E}-A_{I}^{E} M^{I}=0
$$

about which other SMNI nonlinearities bring in multiple minima calculated to be consistent with STM phenomena. In this recent project (Ingber, 2015; Ingber et al., 2014), a Dynamic CM (DCM) model is used as well, wherein the $B_{G^{\prime}}^{G}$ are reset every few epochs of $\tau$.

\subsection{Scale to regions}

Large EEG databases have been used to test scaled SMNI at relatively large regional scales (Ingber, 1997a, 1998). as well as in this project (Ingber, 2015; Ingber et al., 2014). The above Lagrangian $L$ is used across regions, interacting via myelinated fibers represented by $M^{\ddagger E^{\prime}}$ firings.

The context for SMNI describing EEG was developed using the Euler-Lagrange (EL) equations derived from the variational principle associated with SMNI Lagrangians at different scales, giving rise to basic dynamic variables 


$$
\begin{gathered}
\text { Mass }=g_{G G^{\prime}}=\frac{\partial^{2} L}{\partial\left(\partial M^{G} / \partial t\right) \partial\left(\partial M^{G^{\prime}} / \partial t\right)} \\
\text { Momentum }=\Pi^{G}=\frac{\partial L}{\partial\left(\partial M^{G} / \partial t\right)}, \\
\text { Force }=\frac{\partial L}{\partial M^{G}} \\
\mathrm{~F}-\mathrm{ma}=0: \delta L=0=\frac{\partial L}{\partial M^{G}}-\frac{\partial}{\partial t} \frac{\partial L}{\partial\left(\partial M^{G} / \partial t\right)}
\end{gathered}
$$

The momenta $\Pi^{G}$ define Canonical Momenta Indicators (CMI) which were used to advantage in previous SMNI papers fitting EEG (Ingber, 1997a, 1998; Ingber et al., 2014), proving to give superior graphs for analysis to those generated from raw electric potentials $\Phi$. The EL equations are identified with $\mathrm{F}-\mathrm{ma}=0$. This was summarized in a recent paper (Nunez et al., 2013), detailing EL equations at three scales:

\subsubsection{Columnar EL}

Macrocolumnar nearest-neighbor minicolumnar interactions were calculated to include spatial diffusion terms in the Lagrangian defining the conditional probability distribution of mesocolumnar firings. The EL equations were derived from this distribution. Linearization of the space-time EL equations permit the development of stability analyses and dispersion relations in frequency-wave-number space (Ingber, 1982, 1983, 1985b), leading to wave propagation velocities of interactions over several minicolumns, consistent with experiments. This calculation first linearizes the EL, then takes Fourier transforms in space and time variables. A calculation supports observed rotation of images in STM. The earliest studies simply used a driving force $J_{G} M^{G}$ in the Lagrangian to model long-ranged interactions among fibers (Ingber, 1982, 1983).

\subsubsection{Strings EL}

The Lagrangian defining the conditional probability distribution of mesocolumnar firings was transformed to a conditional probability distribution of changes in measured EEG electric potentials. The EL equations were derived from this distribution. This calculation considered one firing variable along the parabolic trough, discussed above, of attractor states being proportional to $\Phi$, the EEG electric potential (Ingber and Nunez, 1990). There exist regions in neocortical parameter space such that the nonlinear string model often used to model EEG is recovered as this EL equation. In this recent study reported here, spline-Laplacian transformations on the $\Phi$ are considered proportional to the firing variables at each electrode site.

\subsubsection{Springs EL}

Macrocolumnar nearest-neighbor minicolumnar interactions were calculated to include spatial diffusion terms in the Lagrangian defining the conditional probability distribution of mesocolumnar firings. The EL equations were derived from this distribution. Some SMNI studies included in calculations regional interactions driving localized columnar activity within these regions (Ingber, 1997a, 1998), instead of the crude model using a driving force $J_{G} M^{G}$ in the Lagrangian as described above. This extension of the above EL equations describes EEG oscillatory behavior supported at these columnar scales across regions (Ingber, 2009; Ingber and Nunez, 2010), which is a model of coupled oscillatory springs across macrocolumns and regions, supported within the string-model envelope described above. 


\subsection{Influence of $q \mathrm{~A}$ on $\mathrm{p}$}

\subsubsection{Classical physics of $\Pi$}

Previous papers have modeled minicolumns as wires which support neuronal firings, due largely from large neocortical excitatory pyramidal cells in layer $\mathrm{V}$ (of six), giving rise to currents which give rise to electric potentials measured as scalp EEG (Ingber, 2011, 2012; Nunez et al., 2013). This gives rise to a magnetic vector potential

$$
\mathbf{A}=\frac{\mu}{4 \pi} \mathbf{I} \log \left(\frac{r}{r_{0}}\right)
$$

which has an insensitive log dependence on distance. In the brain, $\mu \approx \mu_{0}$, where $\mu_{0}$ is the magnetic permeability in vacuum $=4 \pi 10^{-7} \mathrm{H} / \mathrm{m}$ (Henry/meter), where Henry has units of $\mathrm{kg}-\mathrm{m}-\mathrm{C}^{-2}$, which is the conversion factor from electrical to mechanical variables. For oscillatory waves, the magnetic field $\mathbf{B}=\nabla \times \mathbf{A}$ and the electric field $\mathbf{E}=$ $\frac{i c}{\omega} \nabla \times \nabla \times \mathbf{A}$ do not have this log dependence on distance. The magnitude of the current is taken from experimental data on dipole moments $\mathbf{Q}=|\mathbf{I}| z$ where $\hat{\mathbf{z}}$ is the direction of the current $\mathbf{I}$ with the dipole spread over $z$. $\mathbf{Q}$ ranges from $1 \mathrm{pA}-\mathrm{m}=10^{-12} \mathrm{~A}-\mathrm{m}$ for a pyramidal neuron (Murakami and Okada, 2006), to $10^{-9} \mathrm{~A}-\mathrm{m}$ for larger neocortical mass (Nunez and Srinivasan, 2006). These currents give rise to $q \mathbf{A}$ on the order of $10^{-28} \mathrm{~kg}-\mathrm{m} / \mathrm{s}$. $\mathbf{p}$ from one $\mathrm{Ca}^{2+}$ ion in a wave is typically on the order of $10^{-30} \mathrm{~kg}-\mathrm{m} / \mathrm{s}$, and this can be multiplied by the number of ions in a wave, e.g., 100's to 1000 's.

\subsubsection{Quantum physics of $\Pi$}

Previous papers also have detailed quantum calculations of the wave function of $\mathrm{Ca}^{2+}$ waves in the presence of $\mathbf{A}$ (Ingber, 2015; Ingber et al., 2014). The wave function in coordinate space, $\psi(\mathbf{r}, t)$ is

$$
\begin{gathered}
\psi(\mathbf{r}, t)=(2 \pi \hbar)^{-3 / 2} \int_{-\infty}^{\infty} d^{3} \mathbf{p} \phi(\mathbf{p}, t) e^{i \mathbf{p} \cdot \mathbf{r} / \hbar} \\
\psi(\mathbf{r}, t)=\alpha^{-1} e^{-\beta / \gamma-\delta} \\
\alpha=(2 \hbar)^{3 / 2}\left(2 \pi(\Delta \mathbf{p})^{2}\right)^{3 / 4}\left(\frac{i t}{2 m \hbar}-\frac{1}{4(\Delta \mathbf{p})^{2}}\right)^{3 / 2} \\
\beta=\left(\mathbf{r}-\frac{q \mathbf{A} t}{m}-\frac{i \hbar \mathbf{p}_{0}}{2(\Delta \mathbf{p})^{2}}\right)^{2} \\
\gamma=4\left(\frac{i t \hbar}{2 m}+\frac{\hbar^{2}}{4(\Delta \mathbf{p})^{2}}\right) \\
\delta=\frac{\mathbf{p}_{0}^{2}}{4(\Delta \mathbf{p})^{2}}+\frac{i q^{2} \mathbf{A}^{2} t}{2 m \hbar}
\end{gathered}
$$

where $(\Delta \mathbf{p})^{2}$ is the variance of $\mathbf{p}$ in the wave packet, and various properties were calculated and shown to be reasonable in this neocortical context.

If we consider the above wave packet in momentum space, $\phi(\mathbf{p}, t)$ being "kicked" from $\mathbf{p}$ to $\mathbf{p}+\delta \mathbf{p}$, and simply assume that random repeated kicks of $\delta \mathbf{p}$ result in $\left\langle\delta \mathbf{p}>\approx 0\right.$, and each kick keeps the variance $\Delta(\mathbf{p}+\delta \mathbf{p})^{2} \approx \Delta(\mathbf{p})^{2}$, then the overlap integral at the moment $t$ of a typical kick between the new and old state is

$$
\begin{gathered}
<\phi^{*}(\mathbf{p}+\delta \mathbf{p}, t) \mid \phi(\mathbf{p}, t)>=e^{\frac{i k+\rho}{\sigma}} \\
\kappa=8 \delta \mathbf{p} \Delta \mathbf{p}^{2} \hbar m\left(q \mathbf{A}+\mathbf{p}_{0}\right) t-4\left(\delta \mathbf{p} \Delta \mathbf{p}^{2} t\right)^{2}
\end{gathered}
$$




$$
\begin{gathered}
\rho=-(\delta \mathbf{p} \hbar m)^{2} \\
\sigma=8(\Delta \mathbf{p} \hbar m)^{2}
\end{gathered}
$$

where $\phi(\mathbf{p}+\delta \mathbf{p}, t)$ is the normalized wave function in $\mathbf{p}+\delta \mathbf{p}$ momentum space. A crude estimate is obtained of the survival time $A(t)$ and survival probability $p(t)$ (Facchi and Pascazio, 2008),

$$
\begin{gathered}
A(t)=<\phi^{*}(\mathbf{p}+\delta \mathbf{p}, t) \mid \phi(\mathbf{p}, t)> \\
p(t)=|A(t)|^{2}
\end{gathered}
$$

These numbers yield:

$$
<\phi^{*}(\mathbf{p}+\delta \mathbf{p}, t) \mid \phi(\mathbf{p}, t)>=e^{i\left(1.67 \times 10^{-1} t-1.15 \times 10^{-2} t^{2}\right)-1.25 \times 10^{-7}}
$$

Even many repeated kicks do not appreciably affect the real part of $\phi$, and these projections do not appreciably destroy the original wave packet, giving a survival probability per kick as $p(t) \approx \exp \left(-2.5 \times 10^{-7}\right) \approx 1-2.5 \times 10^{-7}$. Both time-dependent phase terms in the exponent are sensitive to time scales on the order of $1 / 10 \mathrm{sec}$, scales prominent in STM and in synchronous neural firings measured by EEG. This suggests that $\mathbf{A}$ effects on $\mathrm{Ca}^{2+}$ wave functions may maximize their influence on STM at frequencies consistent with synchronous EEG during STM by some mechanisms not yet determined.

\subsubsection{PATHTREE}

A sub-project under the current XSEDE.org grant is developing a complex-number version of PATHTREE, an algorithm developed by the author for path integration of financial options (Ingber et al., 2001), also being developed to run on parallel processors under OpenMP. PATHTREE can be used to develop the wave-function above, adding "shocks" to the wave packet to investigate the duration of the wave-packet due to a Zeno/bang-bang effect. This is similar to the use of PATHTREE to include dividends on the underlying asset in financial options. PATHINT is another code developed by the author for path integration used in several disciplines, including the SMNI project (Ingber and Nunez, 1995), but PATHTREE is much faster than PATHINT.

\section{Data}

\subsection{Previous data}

Previous papers in this project used EEG data given to the author circa 1997 (Ingber, 1997b; Zhang et al., 1997a b, 1995). This data was used in other projects (Ingber, 1997a, 1998), as well as in previous calculations in this project (Ingber, 2015; Ingber et al., 2014).

The first use of the data in this project led to examination of multiple graphs to determine differences between no-A and A models (Ingber et al., 2014). This was inconclusive, but leaning to better results with the A model. A second attempt using this data (Ingber, 2015) used statistical measures on the cost functions using the Lagrangian $L$

$$
\{\mathrm{STAT}\}=\{\text { mean, standard-deviation, skewness, kurtosis }\}
$$

applied to each model among the three paradigms presented to each subject, according to whether the subject was classified as $\{\mathrm{a}=$ alcoholic, $\mathrm{c}=$ control (non-alcoholic) $\}$, and according to paradigm $\{1=$ single stimulus, $\mathrm{m}=\mathrm{attempt}$ to match second stimulus to first, $\mathrm{n}=$ no second stimulus matched first $\}$. This also was inconclusive, but leaning to better results with the A model. Further examination showed that several trials had cost functions much larger than the rest, which reasonably could be considered severe outliers skewing results. Rather than "cherry-picking" runs in the data, the decision was made to look for more recent data. 


\subsection{Choice of data}

New data was sought that would satisfy the conditions of the SMNI model, e.g., scalp EEG during STM task, among a reasonable number of subjects, each with a reasonable number or runs that could be divided into Training and Testing sets. A dataset was found with 245 runs across 12 subjects with thousands of epochs per run (Citi et al., 2010; Goldberger et al., 2000). which was downloaded from the

http://physionet.nlm.nih.gov/pn4/erpbci

site, which also contains the useful link

http://www.biosemi.com/download/Cap_coords_all.xls

that was used for spline-Laplacian transformations described below.

The data used was collected during P300 event-related potentials (ERP), in the context of tasks designed to measure changes in shape of the ERP across attentional tasks to non-targets and single and multiple targets via a Donchin speller algorithm. This speller chooses targets from a matrix of 36 characters that the subject must input. Multiple presentations aid in reducing noise of the measured ERP. The authors give rigorous proof and calculations to support the superiority of their Donchin speller algorithm over previous experimental setups (Citi et al., 2010).

\subsection{Conversion to text files}

The data is in European Data Format (EDF) format which contains a lot of information not directly used in this project where simple ascii data is used by fast $\mathrm{C}$ codes to process long optimization sessions. A very useful code was found at the site

http://www.teuniz.net/edf2ascii/

\subsection{Spline-Laplacian transformation}

Arguments have been made, based on a wave-equation analysis of EEG, that Laplacian transformed data are better than the original raw scalp electric potential to represent localized source currents giving rise to EEG (Srinivasan et al., 2006).

Ramesh Srinivasan gave me part of his Matlab code on

$$
\text { http://ssltool.sourceforge.net }
$$

for spline-Laplacian scalp transformations. We then worked together to get this section of code to run under Octave, available from

$$
\text { http://www.gnu.org/software/octave/ }
$$

using the xls file referenced above which contains coordinates of the scalp EEG electrode sites. This smaller code is available at no charge from this author, but any use should reference the ssltool site above.

A typical two-dimensional Laplacian is the weighted difference between four nearest-neighbor points and a central point. Spline fits are smooth-fitting algorithms to a set of discrete points. Here, a spline first fits all (64) sites on the (semi-)circular scalp, generating piecewise smooth curves, so there is actually input from all points before the Laplacian is taken. Smoothing data points before taking derivatives, e.g., expanding the number of points used to calculate the Laplacian (Lynch, 1992), is a good counter-measure to introducing noise whenever derivative operations are used on noisy data, as would occur if numerical Laplacians were used. In this case the spline fits develops an analytic function with smooth Laplacians. The Laplacian permits a better localization of sources, since there is a general diffusion of electric potential especially due to the scalp and skull. There is dependence on the spline fits to accomplish a lot but still remaining faithful to the data, e.g., properly representing distant regions of cortex included at each region-site, but studies support this approach as better than others (Srinivasan et al., 2006). 
After conversion to text files, each run had a data file of about 70,000 lines, each representing about $0.5 \mathrm{msec}$. Using Octave, the spline-Laplacian transformation was applied across 64 columns representing electrode sites for each of these lines of data across all 245 runs.

When applied to P300 data here, the spline-Laplacian transformed data were similar, but more tightly grouped, than the original scalp electric potential data.

\subsection{Coarse-graining time resolution}

The SMNI model is bast on time resolutions refractory period of $\tau \approx 5 \mathrm{msec}$ is taken to lie between an absolute refractory period of $\approx 1 \mathrm{msec}$, during which another action potential cannot be initiated, and a relative refractory period of $\approx 0.5--10 \mathrm{msec}$. Therefore this data was then coarse-grained with a moving average of 4 epochs, with about 17,500 lines representing time resolutions of about $2 \mathrm{msec}$. The use of a narrow moving average to reduce noise was used effectively in copula risk management of financial markets, as discussed in a generalized approach to applications of SMNI (Ingber, 2007), as originally reported in the finance literature (Litterman and Winkelmann, 1998). This method worked better in these contexts than more sophisticated algorithms developed using random matrices (Laloux et al., 1999).

\subsection{Cumulative graphs select time window}

All 245 runs across all epochs were placed on one graph. Consistent with the experimental design, this showed several regions of cumulative high amplitudes during which STM tasks were performed by the 12 subjects. A region of continuous high amplitude was chosen of 2561 lines representing times from 17 to 22 secs.

\subsection{Cumulative graphs select electrode sites}

For each of 64 electrode sites, cumulative graphs over all 245 runs for all subjects $\{\mathrm{s} 01 \ldots$ s12\} were examined to select sites with obvious strong signals. The four sites $\{05-\mathrm{F} 3,37-\mathrm{AFz}, 40-\mathrm{F} 4,48-\mathrm{Cz}\}$ showed similar strong signals with two main peaks and one main valley. Other sites had varying degrees of much larger spreads of scattered data. Figure 1 is an example comparison from electrode site 05-F3, using cumulative data over 20 subjects, each with $20+$ runs, displaying a graph of the electric potential data measured at one electrode across all subjects across all runs, versus a graph of the moving-averaged (discussed above) spline-Laplacian data. The reduction of noise is due to the moving average and spline algorithms, and the general shape change is due to the Laplacian algorithm applied to the entire set of 64 electrodes.

\subsection{All graphs}

A supplemental file contains graphs relevant to these calculations,

https://www.ingber.com/smni16_large-scale_molecular_EEGgraphs.pdf

The first three pages are a pdf rendering of the 64-channel page in

http://www.biosemi.com/download/Cap_coords_all.xls

The pdf file contains are two sets of graphs, side by side, the raw potentials and the spline-Laplacian transformed potentials:

\subsubsection{Raw Potential}

This set of 64 graphs contains all 245 runs from all 12 subjects of raw electric potential data for each electrode site, within the time ranges discussed in this paper. 


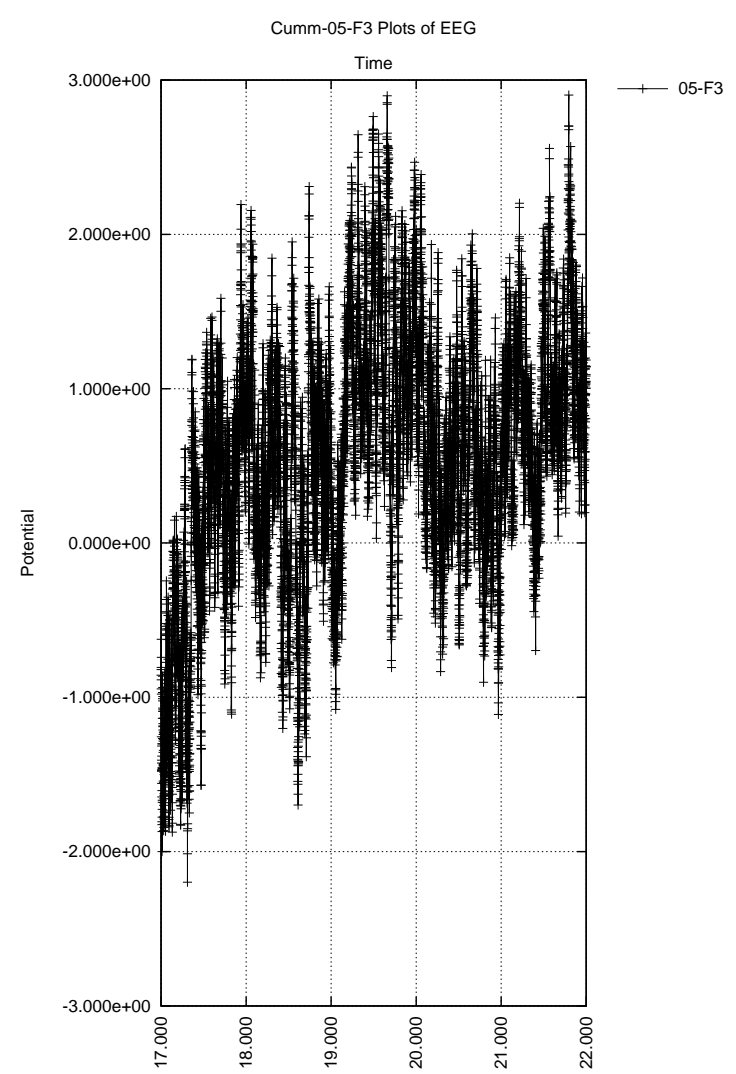

(a) electric potential

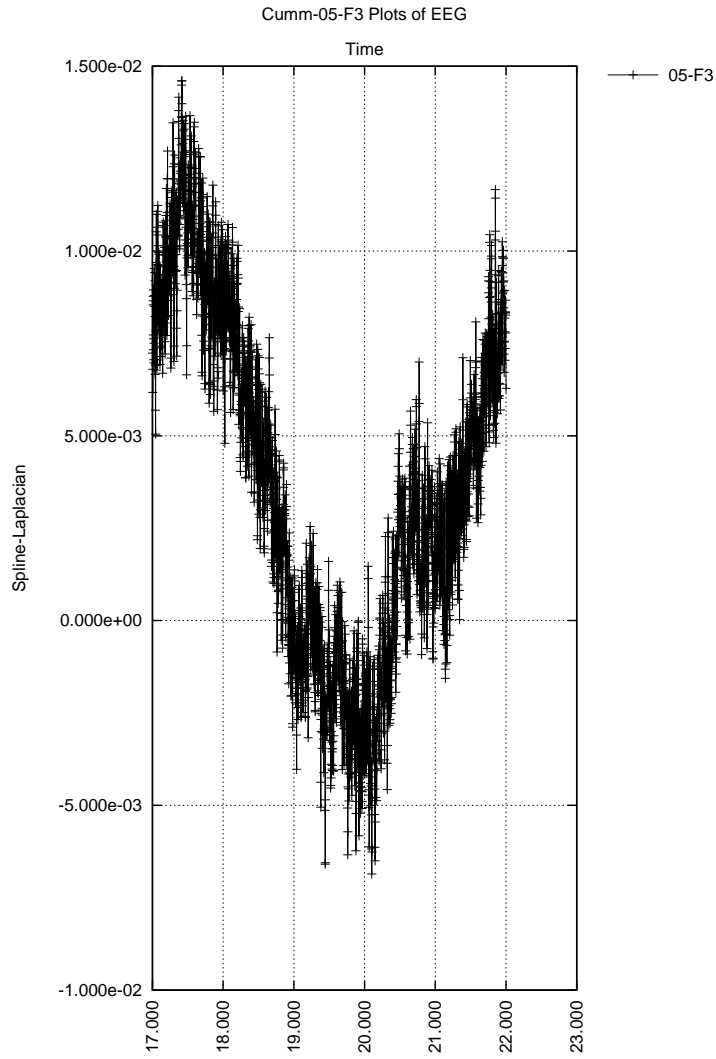

(b) spline-Laplacian

Figure 1: Cumulative data over 20 subjects from EEG electrode site 05-F3 is displayed: (a) Raw electric potential data. (b) Averaged spline-Laplacian data. 


\subsubsection{Spline-Laplacian}

This set of 64 graphs contains all 245 runs from all 12 subjects of spline-Laplacian moving-averaged transformed data for each electrode site, within the time ranges discussed in this paper.

\section{Fitting Theory To Data}

\subsection{Towards a zero-fit-parameter model}

In keeping more strictly with the SMNI zero-fit-parameter philosophy, fits to data minimize the number of parameters to more strongly test theory. Here, parameters are just the strength of regional connections. Simple functional contributions of data (spline-Laplacian transformations of scalp electric potential) dependence, drift-dependent as discussed below, to $B_{G^{\prime}}^{G}$ background were taken to be $1 / 2$ of the no-A model. No additional parameters are considered necessary to make the strong point that DSM is a better fit to the data than CM, both of which are better than no CM. Therefore, there also are no parameters scaling electric potentials to mesocolumnar firings as in previous papers, but rather the data was used to determine scales as discussed below. This was done in the context of using spline-Laplacian transformations, to more strictly enforce the correspondence between the transformed data and the mesocolumnar firings, which may be electrode as well as subject dependent (due to difference in skull/scalp properties, etc.).

\subsection{Refine data}

Instead of scaling columnar firings with parameters, for each subject, for all runs using the chosen section of splineLaplacian data, the maximum and minimum values of data were used to scale maximum and minimum firing, $\pm N^{E}$ within each region, i.e., in each region, each spline-Laplacian data point $s l$ was scaled to firing states by the factor $M_{s l}$,

$$
\begin{gathered}
s l>0: M_{s l}=\min \left[N^{E}, s l * N^{E} /(\max [\max (s l),|\min (s l)|])\right] \\
s l<0: M_{s l}=\max \left[-N^{E}, s l * N^{E} /(\max [\max (s l),|\min (s l)|])\right]
\end{gathered}
$$

\subsection{A contribution to synaptic background}

The waves depend on aggregates of their $\boldsymbol{\Pi}=\mathbf{p}+q \mathbf{A}$ dynamics. E.g., this can be modeled as a Taylor expansion in $|\mathbf{A}|$,

$$
B_{G^{\prime}}^{G} \rightarrow B_{G^{\prime}}^{G}+\mathbf{A}{B^{\prime}}_{G^{\prime}}^{G}, B_{E^{\prime}}^{\ddagger E}=B_{E^{\prime}}^{\ddagger E}+\mathbf{A}{B^{\prime}}_{E^{\prime}}^{\ddagger E}
$$

For the A model, only for $B_{G^{\prime}}^{\prime E}$ was added with a factor of the SMNI drift. The contribution of the product $\mathbf{A}{B^{\prime}}_{G^{\prime}}$, is taken to be a drift factor multiplied by a factor of $B_{G^{\prime}}^{G}$. The drift for each region is not calculated from any trend of the data, but from the SMNI nonlinear drift $g^{G}$, as a moving average over the last 3 epochs representing about 6 msec. The moving-averaged drift numerically fell between -0.5 and 0.5 .

\subsection{Summary of proportionalities}

In summary,

$$
M^{G} \propto \mathbf{I}
$$

$\mathrm{EEG} \propto \mathbf{I}$

$$
\mathbf{A} \propto \mathbf{I}
$$




$$
\begin{gathered}
{B^{\prime}}_{G^{\prime}}^{E} \propto\left[\mathrm{Ca}^{2+}\right] \\
{\left[\mathrm{Ca}^{2+}\right] \propto \mathbf{A}}
\end{gathered}
$$

where the last proportionality applies to the influence of $\mathbf{A}$ on free $\left[\mathrm{Ca}^{2+}\right]$ at synapses via $\boldsymbol{\Pi}=\mathbf{p}+q \mathbf{A}$.

\subsection{ASA}

The author's Adaptive Simulated Annealing (ASA) code (Ingber, 1993) is used for all optimization to fit SMNI parameters to EEG data.

The main ASA OPTIONS that were turned on to tune the optimization were:

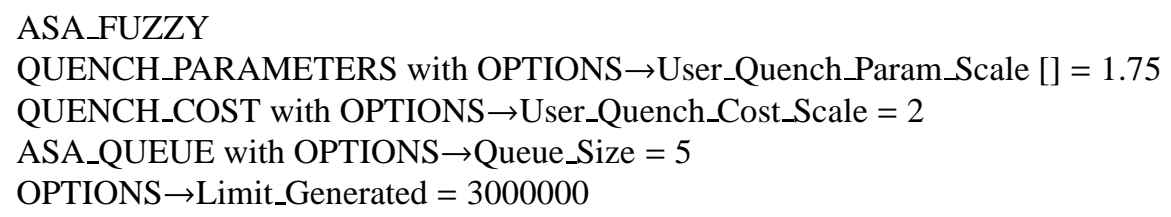

The QUENCH OPTIONS were set to get good convergence to minima, tested with longer runs without the QUENCHing within 1,000,000 generated states.

The simplex code, contained in the asa_usr.c file of the ASA code, was run after ASA, permitting up to 5000 additional generated states. No better solutions were obtained within max-min bounds of parameters.

Runs were performed on The Extreme Science and Engineering Discovery Environment platforms, as described at XSEDE.org. XSEDE clock time running 24 nodes under MPI, i.e., independent runs for each of 12 subjects, each subject run with A model and no-A model, was $37 \mathrm{hrs}$ on Stampede (Texas Advanced Computing Center (TACC) Dell PowerEdge C 8220 Cluster with Intel Xeon Phi coprocessors) $=888$ CPU hrs per run, or 48 hrs on Gordon (San Diego Supercomputer Center (SDSC) Appro with 8-core, 2.6-GHz Intel Sandy Bridge processors) $=1152 \mathrm{CPU}$ hrs per run.

\subsection{Compare Testing with Training}

For each of the 12 subjects, it was possible to find 10 Training runs and 10 Testing runs. Comparison with switched runs for Training and Testing reveals some subjects with modest outlier runs; i.e., if they were consistent across runs, then their Training cost functions should be less than their Testing cost functions. The Table below presents the results. As can be seen, the $\mathbf{A}$ model clearly outperformed the no-A model. Note that cost functions with an $|\mathbf{A}|$ model are much worse that either the A model or the no-A model. Runs with different signs on the drift and on the absolute value of the drift also gave much higher cost functions that the $\mathbf{A}$ model.

\section{Conclusion}

An SMNI model has been developed to calculate coupling of molecular scales of $\mathrm{Ca}^{2+}$ wave dynamics with $\mathbf{A}$ fields developed at macroscopic regional scales measured by coherent neuronal firing activity measured by scalp EEG during STM tasks. This requires crossing molecular, microscopic (synaptic and neuronal), mesoscopic (minicolumns and macrocolumns), and macroscopic regional scales.

Considerations of both classical and quantum physics give predictions of the influence of $\mathbf{A}$ on the momenta of $\mathrm{Ca}^{2+}$ waves during STM processing as measured by scalp EEG. Since the spatial scales of $\mathrm{Ca}^{2+}$ wave and macro-EEG are quite disparate, an experiment would have to be able to correlate both scales in time scales on the order of tens of milliseconds.

This study is robust against much theoretical modeling, as experimental data is used wherever possible. The theoretical construct of the canonical momentum $\boldsymbol{\Pi}=\mathbf{p}+q \mathbf{A}$ is firmly entrenched in classical and quantum mechanics. 
Table 1: Column 1 is the subject number; the other columns are cost functions. Columns 2 and 3 are no-A model's Training (TR0) and Testing (TE0). Columns 4 and 5 are A model's Training (TRA)and Testing (TEA). Columns 4 and 5 are no-A model's Training (TRs0) and Testing (TEs0), with switched runs. Columns 6 and 7 are A model's Training (TRsA)and Testing (TEsA), with switched runs. Columns 8 and 9 are $|\mathbf{A}|$ model's Training (TR $|\mathbf{A}|$ ) and Testing (TE|A|).

\begin{tabular}{|c|cc|cc|cc|cc|cc|}
\hline Sub & TR0 & TE0 & TRA & TEA & TRs0 & TEs0 & TRsA & TEsA & TR $|\mathbf{A}|$ & TE $|\mathbf{A}|$ \\
\hline s01 & 85.61 & 121.4 & 62.08 & 97.31 & 120.3 & 86.85 & 96.62 & 62.90 & 98.17 & 132.3 \\
s02 & 70.66 & 51.27 & 52.90 & 36.56 & 51.02 & 70.81 & 36.45 & 53.05 & 96.92 & 79.31 \\
s03 & 61.26 & 79.58 & 43.12 & 55.63 & 78.96 & 61.43 & 55.24 & 43.22 & 83.74 & 104.5 \\
s04 & 52.25 & 64.12 & 34.46 & 46.58 & 63.49 & 53.06 & 45.98 & 35.00 & 70.77 & 83.36 \\
s05 & 67.20 & 72.22 & 47.29 & 51.44 & 71.30 & 67.78 & 51.14 & 47.64 & 85.52 & 88.36 \\
s06 & 84.46 & 69.44 & 64.89 & 45.17 & 68.88 & 84.58 & 44.72 & 64.98 & 96.90 & 82.72 \\
s07 & 68.60 & 78.62 & 49.67 & 56.87 & 78.37 & 68.76 & 56.77 & 49.76 & 87.00 & 95.64 \\
s08 & 47.09 & 44.16 & 34.23 & 34.89 & 43.46 & 47.89 & 34.76 & 34.57 & 72.11 & 68.90 \\
s09 & 47.52 & 25.22 & 39.19 & 16.47 & 24.96 & 48.06 & 16.29 & 39.74 & 85.37 & 66.76 \\
s10 & 53.10 & 33.33 & 40.35 & 22.51 & 33.10 & 53.81 & 22.39 & 40.75 & 75.93 & 66.75 \\
s11 & 43.91 & 51.15 & 33.21 & 37.64 & 50.93 & 44.38 & 37.52 & 33.50 & 70.90 & 87.31 \\
s12 & 45.71 & 45.20 & 30.99 & 31.58 & 44.85 & 46.07 & 31.45 & 31.15 & 65.15 & 70.59 \\
\hline
\end{tabular}

Previous calculations appeared to suffer from severe outliers in the EEG data used to fit the SMNI models. Therefore new data was sought and after a selection was made, care was taken to select sections of data appropriate to the SMNI model, e.g., during P300 task, moving averages consistent with SMNI time scales, etc. New fits indeed show that the $\mathbf{A}$ is a much better fit to the data than the no-A model.

\section{Acknowledgment}

I thank the National Science Foundation's Extreme Science and Engineering Discovery Environment (XSEDE.org), for three supercomputer grants since February 2013, "Electroencephalographic field influence on calcium momentum waves", one under PHY130022 and two under TG-MCB140110. I thank Ramesh Srinivasan for giving me part of his Matlab code for spline-Laplacian scalp transformations. 


\section{References}

Agulhon, C., Petravicz, J., McMullen, A., Sweger, E., Minton, S., Taves, S., Casper, K., Fiacco, T., McCarthy, K., 2008. What is the role of astrocyte calcium in neurophysiology? Neuron 59, 932-946.

Asher, J., 2012. Brain's code for visual working memory deciphered in monkeys NIH-funded study. Technical Report NIH Press Release. NIH. Bethesda, MD. http://www.nimh.nih.gov/news/science-news/2012/in-sync-brain-waves-hold-memory-of-objects-just-seen.shtml

Atmanspacher, H., 2015. Quantum approaches to consciousness, in: Zalta, E. (Ed.), The Stanford Encyclopedia of Philosophy. Stanford U, Palo Alto, p. 1. http://plato.stanford.edu/archives/sum2015/entries/qt-consciousness

Beck, F., $2008 . \quad$ Synaptic quantum tunnelling in brain activity. $\quad$ Neuroquantology 6, $140-151$. http://dx.doi.org/10.14704/nq.2008.6.2.168

Cheng, K., 1972. Quantization of a general dynamical system by Feynman's path integration formulation. Journal of Mathematical Physics 13, 1723-1726.

Citi, L., Poli, R., Cinel, C., 2010. Documenting, modelling and exploiting P300 amplitude changes due to variable target delays in Donchin's speller. Journal of Neural Engineering 7, 1-21. http://dx.doi.org/10.1088/1741-2560/7/5/056006

Facchi, P., Lidar, D., Pascazio, S., 2004. Unification of dynamical decoupling and the quantum zeno effect. Physical Review A 69, 1-6.

Facchi, P., Pascazio, S., 2008. Quantum zeno dynamics: mathematical and physical aspects. Journal of Physics A 41, 1-45.

Fiacco, T., McCarthy, K., 2004. Intracellular astrocyte calcium waves in situ increase the frequency of spontaneous AMPA receptor currents in CA1 pyramidal neurons. Journal of Neuroscience 24, 722-732.

Giacosa, G., Pagliara, G., 2014. Quantum zeno effect by general measurements. Physical Review A 052107, 1-5.

Goldberger, A., Amaral, L., Glass, L., Hausdorff, J., Ivanov, P., Mark, R., Mietus, J., Moody, G., Peng, C.K., Stanley, H., 2000. PhysioBank, PhysioToolkit, and PhysioNet: components of a new research resource for complex physiologic signals. Circulation 101, e215-e220. http://circ.ahajournals.org/cgi/content/full/101/23/e215

Ingber, L., 1982. Statistical mechanics of neocortical interactions. i. basic formulation. Physica D 5, 83-107. http://www.ingber.com/smni82_basic.pdf

Ingber, L., 1983. Statistical mechanics of neocortical interactions. dynamics of synaptic modification. Physical Review A $28,395-416$. http://www.ingber.com/smni83_dynamics.pdf

Ingber, L., 1984. Statistical mechanics of neocortical interactions. derivation of short-term-memory capacity. Physical Review A 29, $3346-3358$. http://www.ingber.com/smni84_stm.pdf

Ingber, L., 1985a. Statistical mechanics of neocortical interactions: Stability and duration of the 7+-2 rule of short-term-memory capacity. Physical Review A 31, 1183-1186. http://www.ingber.com/smni85_stm.pdf

Ingber, L., 1985b. Statistical mechanics of neocortical interactions. EEG dispersion relations. IEEE Transactions in Biomedical Engineering 32, 91-94. http://www.ingber.com/smni85_eeg.pdf

Ingber, L., 1993. Adaptive Simulated Annealing (ASA). Technical Report Global optimization C-code. Caltech Alumni Association. Pasadena, CA. http://www.ingber. com/\#ASA-CODE

Ingber, L., 1994. Statistical mechanics of neocortical interactions: Path-integral evolution of short-term memory. Physical Review E 49, $4652-4664$. http://www.ingber.com/smni94_stm.pdf

Ingber, L., 1997a. Statistical mechanics of neocortical interactions: Applications of canonical momenta indicators to electroencephalography. Physical Review E 55, 4578-4593. http://www.ingber.com/smni97_cmi.pdf

Ingber, L., 1997b. EEG Database. UCI Machine Learning Repository, Irvine, CA. http://archive .ics .uci.edu/ml/datasets/EEG+Database

Ingber, L., 1998. Statistical mechanics of neocortical interactions: Training and testing canonical momenta indicators of EEG. Mathematical Computer Modelling 27, 33-64. http://www.ingber.com/smni98_cmi_test.pdf

Ingber, L., 2007. Ideas by statistical mechanics (ISM). Journal Integrated Systems Design and Process Science 11, 31-54. Special Issue: Biologically Inspired Computing.

Ingber, L., 2009. Statistical mechanics of neocortical interactions: Nonlinear columnar electroencephalography. NeuroQuantology Journal 7 , 500-529. http://www .ingber.com/smni09_nonlin_column_eeg.pdf

Ingber, L., 2011. Computational algorithms derived from multiple scales of neocortical processing, in: Pereira, Jr., A., Massad, E., Bobbitt, N. (Eds.), Pointing at Boundaries: Integrating Computation and Cognition on Biological Grounds. Springer, New York, pp. 1-13. Invited Paper. http://www.ingber.com/smni11_cog_comp.pdfandhttp://dx.doi.org/10.1007/s12559-011-9105-4

Ingber, L., 2012. Columnar EEG magnetic influences on molecular development of short-term memory, in: Kalivas, G., Petralia, S. (Eds.), ShortTerm Memory: New Research. Nova, Hauppauge, NY, pp. 37-72. Invited Paper.http://www.ingber.com/smni11_stm_scales.pdf

Ingber, L., 2015. Calculating consciousness correlates at multiple scales of neocortical interactions, in: Costa, A., Villalba, E. (Eds.), Horizons in Neuroscience Research. Nova, Hauppauge, NY, pp. 153-186. ISBN: 978-1-63482-632-7. Invited paper. http://www.ingber.com/smni15_calc_conscious.pdf

Ingber, L., Chen, C., Mondescu, R., Muzzall, D., Renedo, M., 2001. Probability tree algorithm for general diffusion processes. Physical Review E 64, 056702-056707. http://www.ingber.com/path01_pathtree.pdf

Ingber, L., Nunez, P., 1990. Multiple scales of statistical physics of neocortex: Application to electroencephalography. Mathematical Computer Modelling 13, 83-95.

Ingber, L., Nunez, P., 1995. Statistical mechanics of neocortical interactions: High resolution path-integral calculation of short-term memory. Physical Review E 51, 5074-5083. http://www.ingber.com/smni95_stm.pdf

Ingber, L., Nunez, P., 2010. Neocortical dynamics at multiple scales: EEG standing waves, statistical mechanics, and physical analogs. Mathematical Biosciences 229, 160-173. http://www .ingber.com/smni10_multiple_scales.pdf

Ingber, L., Pappalepore, M., Stesiak, R., 2014. Electroencephalographic field influence on calcium momentum waves. Journal of Theoretical 
Biology 343, 138-153. http://www.ingber.com/smni14_eeg_ca.pdfandhttp://dx.doi.org/10.1016/j.jtbi.2013.11.002

Innocenti, B., Parpura, V., Haydon, P., 2000. Imaging extracellular waves of glutamate during calcium signaling in cultured astrocytes. Journal of Neuroscience 20, 1800-1808.

Kozlowski, W., Caballero-Benitez, S., Mekhov, I., 2015. Non-hermitian dynamics in the quantum Zeno limit. Technical Report arXiv:1510.04857 [quant-ph]. U Oxford. Oxford, UK.

Laloux, L., Cizeau, P., Bouchaud, J., Potters, M., 1999. Noise dressing of financial correlation matrices. Physical Review Letters 83, 1467-1470.

Langouche, F., Roekaerts, D., Tirapegui, E., 1982. Functional Integration and Semiclassical Expansions. Reidel, Dordrecht, The Netherlands.

Litterman, R., Winkelmann, K., 1998. Estimating covariance matrices. Technical Report Report. Goldman Sachs. New York.

Lynch, R., 1992. Fundamental solutions of nine-point discrete laplacians. Applied Numerical Mathematics 10, 325-334.

McKemmish, L., Reimers, J., McKenzie, R., Mark, A., Hush, N., 2009. Penrose-hameroff orchestrated objective-reduction proposal for human consciousness is not biologically feasible. Physical Review E 80,1-6. http://link.aps.org/doi/10.1103/PhysRevE.80.021912

Mitterauer, B., Kofler-Westergren, B., 2011. Possible effects of synaptic imbalances on oligodendrocyte-axonic interactions in schizophrenia: A hypothetical model. Frontiers in Psychiatry 2, 1-13. http://doi.org/10.3389/fpsyt.2011.00015

Murakami, S., Okada, Y., 2006. Contributions of principal neocortical neurons to magnetoencephalography and electroencephalography signals. Journal of Physiology 575, 925-936.

Nunez, P., Srinivasan, R., Ingber, L., 2013. Theoretical and experimental electrophysiology in human neocortex: Multiscale correlates of conscious experience, in: Pesenson, M. (Ed.), Multiscale Analysis and Nonlinear Dynamics: From genes to the brain. Wiley, New York, pp. $149-178$. http://dx.doi.org/10.1002/9783527671632.ch06

Nunez, P.L., Srinivasan, R., 2006. Electric Fields of the Brain: The Neurophysics of EEG, 2nd Ed. Oxford University Press, London.

Patil, Y., Chakram, S., Vengalattore, M., 2015. Measurement-induced localization of an ultracold lattice gas. Physical Review Letters 115, 1-5. http://link.aps.org/doi/10.1103/PhysRevLett.115.140402

Pereira, Jr., A., Furlan, F.A., 2009. On the role of synchrony for neuron-astrocyte interactions and perceptual conscious processing. Journal of Biological Physics 35, 465-480.

Ross, W., 2012. Understanding calcium waves and sparks in central neurons. Nature Reviews Neuroscience 13, $157-168$.

Salazar, R., Dotson, N., Bressler, S., Gray, C., 2012. Content-specific fronto-parietal synchronization during visual working memory. Science 338, 1097-1100. http://dx.doi.org/10.1126/science.1224000

Scemes, E., Giaume, C., 2006. Astrocyte calcium waves: What they are and what they do. Glia 54, 716-725. http://dx.doi.org/10.1002/glia.20374

Shaw, G., Vasudevan, R., 1974. Persistent states of neural networks and the random nature of synaptic transmission. Mathematical Biosciences 21, 207-218.

Srinivasan, R., Winter, W., Nunez, P., 2006. Source analysis of EEG oscillations using high-resolution EEG and MEG. Progress Brain Research $159,29-42$.

Volterra, A., Liaudet, N., Savtchouk, I., 2014. Astrocyte Ca2+ signalling: an unexpected complexity. Nature Reviews Neuroscience 15, 327-335.

Wu, S., Wang, L., Yi, X., 2012. Time-dependent decoherence-free subspace. Journal of Physics A 405305, 1-11.

Zhang, P., Ai, Q., Li, Y., Xu, D., Sun, C., 2014. Dynamics of quantum zeno and anti-zeno effects in an open system. Science China Physics, Mechanics Astronomy 57, 194-207. http://dx.doi.org/10.1007/s11433-013-5377-x

Zhang, X., Begleiter, H., Porjesz, B., 1997a. Do chronic alcoholics have intact implicit memory? an ERP study. Electroencephalography Clinical Neurophysiology 103, 457-473.

Zhang, X., Begleiter, H., Porjesz, B., Litke, A., 1997b. Electrophysiological evidence of memory impairment in alcoholic patients. Biological Psychiatry 42, 1157-1171.

Zhang, X., Begleiter, H., Porjesz, B., Wang, W., Litke, A., 1995. Event related potentials during object recognition tasks. Brain Research Bulletin $38,531-538$.

Zhou, D., Mowrey, D., Tang, P., Xu, Y., 2015. Percolation model of sensory transmission and loss of consciousness under general anesthesia. Physical Review Letters 115, 1-6. http://link.aps.org/doi/10.1103/PhysRevLett.115.108103 